\title{
Experiments on wake structures behind a microparticle in a magnetized plasma flow
}

Cite as: Phys. Plasmas 25, 073703 (2018); https://doi.org/10.1063/1.5040838

Submitted: 21 May 2018 . Accepted: 21 June 2018 . Published Online: 10 July 2018

Hendrik Jung (D), Franko Greiner (D), Alexander Piel, and Wojciech J. Miloch (D)

COLLECTIONS

F This paper was selected as Featured
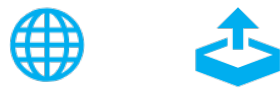

\section{ARTICLES YOU MAY BE INTERESTED IN}

Charging of an irregularly shaped particle in the sheath of an rf plasma Physics of Plasmas 25, 073702 (2018); https://doi.org/10.1063/1.5038183

Dust density waves in a dc flowing complex plasma with discharge polarity reversal Physics of Plasmas 25, 083705 (2018); https://doi.org/10.1063/1.5040417

A simple method of dust charge estimation using an externally applied oscillating electric field

Physics of Plasmas 25, 073701 (2018); https://doi.org/10.1063/1.5037020

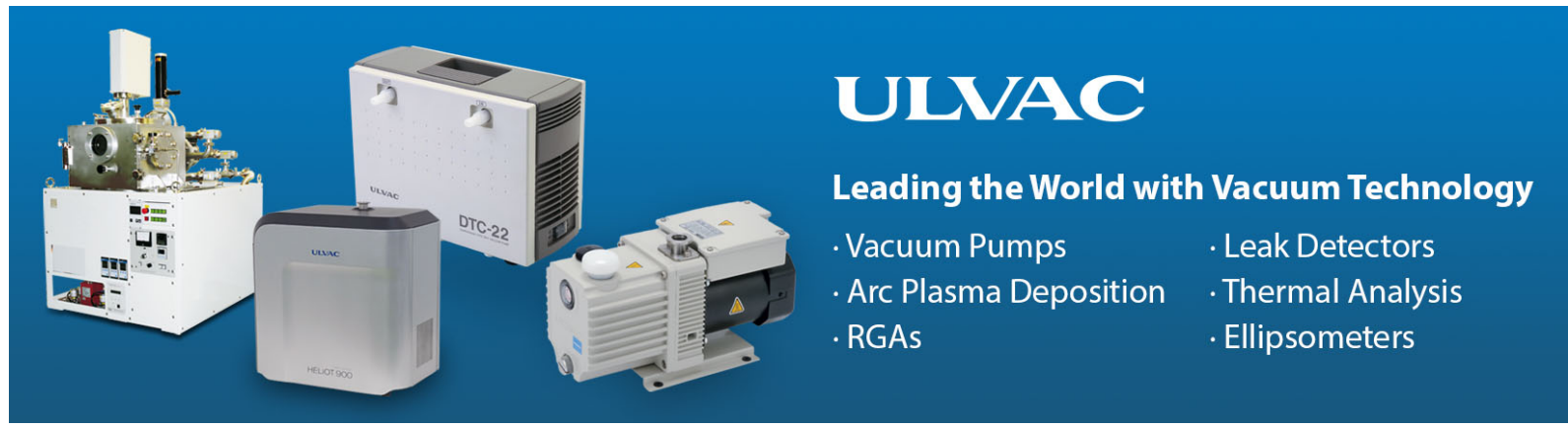




\title{
Experiments on wake structures behind a microparticle in a magnetized plasma flow
}

\author{
Hendrik Jung, ${ }^{1, a)}$ Franko Greiner, ${ }^{1}$ Alexander Piel, ${ }^{1}$ and Wojciech J. Miloch ${ }^{2}$ \\ ${ }^{1}$ IEAP, Christian-Albrechts-Universität, D-24098 Kiel, Germany \\ ${ }^{2}$ Department of Physics, University of Oslo, P.O. Box 1048, Blindern, 0317 Oslo, Norway
}

(Received 21 May 2018; accepted 21 June 2018; published online 10 July 2018)

\begin{abstract}
The wake behind a spherical microparticle in a magnetized ion flow is studied experimentally by analyzing the arrangement of a pair of particles. It is shown that there are two stable particle arrangements at intermediate magnetic inductions, whereas only oblique (horizontal) particle configurations are found at the highest magnetic field. Self-consistent collisional molecular dynamics simulations of the particle system show that the underlying mechanism of these arrangements is the weakening of attractive wake forces by the increasing magnetic field. Plasma instabilities provide a trigger for the onset of the transition between the two different arrangements. Furthermore, the course of the transition is qualitatively explained by the charge variation of the downstream particle in the wake of the upstream particle. In addition, a thorough analysis of the sheath by means of particle-in-cell simulations in combination with particle resonance measurements yields consistent values of the particle mass and charge, as well as the levitating electric field and ion flow velocity. Published by AIP Publishing. https://doi.org/10.1063/1.5040838
\end{abstract}

\section{INTRODUCTION}

In laboratory complex plasmas, experiments with a small number of microparticles are generally performed in the plasma sheath. The strong sheath electric field that provides the levitation of the particle also accelerates ions to supersonic flows, and an ion wake is formed downstream of the particle. $^{1-3}$ In the unmagnetized case, a characteristic feature of the wake is an accumulation of ions due to focusing of the ion flow by the upstream particle. The associated wake electric fields give rise to intricate interactions of microparticles: While the interaction of two particles arranged perpendicularly to the flow can be described by the common Yukawa (Debye-Hückel) potential, ${ }^{4}$ the situation in which the particles are aligned parallel to the flow is inhomogeneous and nonlinear. The enhanced ion density in the focus region provides horizontal attractive forces on other microparticles ${ }^{2,3,5}$ and leads to a reduction of the negative charge of a downstream particle. $^{6-9}$ The present state of the understanding of wakes is summarized in recent reviews. ${ }^{10,11}$

In contrast to simulations ${ }^{6,12-16}$ where potential and density distributions can be analyzed directly, wake structures can only be investigated experimentally by indirect methods, e.g., by their influence on other microparticles. Typical methods for the measurement of the spatial properties of the plasma as Langmuir probe measurements are not applicable here due to the size of these wake structures being smaller than the Debye length. Spectroscopic methods like laserinduced fluorescence (LIF) have been used to measure ion wake effects around a $10 \mathrm{~mm}$ sphere in a plasma, ${ }^{17}$ and the spatial resolution needed for the investigation of a microparticle remains an unresolved challenge. Information on the wake structure of a microparticle has been obtained by

\footnotetext{
${ }^{\text {a)} E l e c t r o n i c ~ m a i l: ~ h j u n g @ p h y s i k . u n i-k i e l . d e ~}$
}

analyzing the charge of another particle inside this wake $^{7,9,18,19}$ or by measuring the wake-induced non-reciprocal particle interaction forces $2,3,7,9,18,19$ by evaluating the dynamical response of the particles to external excitations.

The coexistence of vertically aligned and horizontal pairs of particles was demonstrated in Ref. 20. By analyzing (the transitions between) these states and the underlying instability mechanisms, ${ }^{21,22}$ information on the wake charge $^{20}$ and the spatial extent of the wake of the upstream particle is gained, ${ }^{23,24}$ and the oscillatory modes can be characterized by measuring the velocity correlation. ${ }^{25,26}$ By pushing the downstream particle of a stable, vertically aligned particle pair with a strong laser beam, the pair can be brought into a meta-stable oblique arrangement, showing the attractive character of the wake and providing information on its spatial extent. ${ }^{24,27}$

The trajectory analysis of low-energy collisions between two particles of different masses provides full $2 \mathrm{~d}$ information of the wake potential. ${ }^{28,29}$ Such investigations can be further improved by taking the charge variation in the wake into account: By means of plasma-inherent etching processes, the mass of the downstream particle and, as a consequence, the distance between two particles have been continuously reduced. In this way, by measuring the charges and interaction forces, full information on the longitudinal wake structure of a single particle in the plasma sheath was obtained. ${ }^{9}$

While there is a broad range of studies concerning these wake structures in the unmagnetized case, there is a lack of experimental observations for strongly magnetized systems. Magnetic inductions of $B \geq 0.5 \mathrm{~T}$ are of great interest since this is the regime where the Lorentz force on the ions becomes competitive with the other major forces in the system, such as inertial force, coupling force, and damping force. First experimental investigations ${ }^{30}$ and calculations in the linear response (LR) approximation ${ }^{31-33}$ indicate the 
damping of the wake potential. Self-consistent particle-incell (PIC) simulations ${ }^{34,35}$ and molecular dynamics (MD) simulations ${ }^{36,37}$ have shown the appearance of ion shadows (regions of ion depletion) and the vanishing of the attractive interaction forces at strong magnetic fields.

The aim of this paper is to investigate the modification of the wake by strong magnetic fields. For this purpose, the arrangement of a pair of microparticles in the plasma sheath is analyzed at different states of plasma magnetization. We focus on characteristic changes of the particle configuration and relate them to the magnetic-field-induced modification of the wake and the corresponding interparticle forces.

In Sec. II, we describe the experimental setup and characterize the plasma and the microparticles used in the experiment. The results of the experiments at magnetic fields are presented in Sec. III. In Sec. IV, the experimental results are discussed in the context of MD simulations. Conclusions are given in Sec. V.

\section{STRONGLY MAGNETIZED PLASMA SHEATH}

\section{A. Experimental setup and plasma filamentation}

A sketch of the setup is shown in Fig. 1. The experiments are performed in a symmetric, capacitively coupled radio-frequency (rf) discharge. Argon gas is used at a pressure of $p=10 \mathrm{~Pa}$, and the $\mathrm{rf}$ power is $P \approx 5 \mathrm{~W}$, typically. Our test particles are hollow glass spheres (microballoons ${ }^{38}$ ) with outer radii of $a \in[10,12.5] \mu \mathrm{m}$ (see Sec. II B).

We use a vertical pair of glass microballoons which levitates in the plasma sheath over the lower electrode. Here, the (time-averaged) vertical sheath electric field $\langle E\rangle$ is strong enough to compensate the force of gravity and hence levitates the particles according to the Millikan condition

$$
q\langle E\rangle=m g,
$$

where $g$ is the gravitational acceleration and $q$ and $m$ are the particle charge and mass, respectively. Note that the neglect

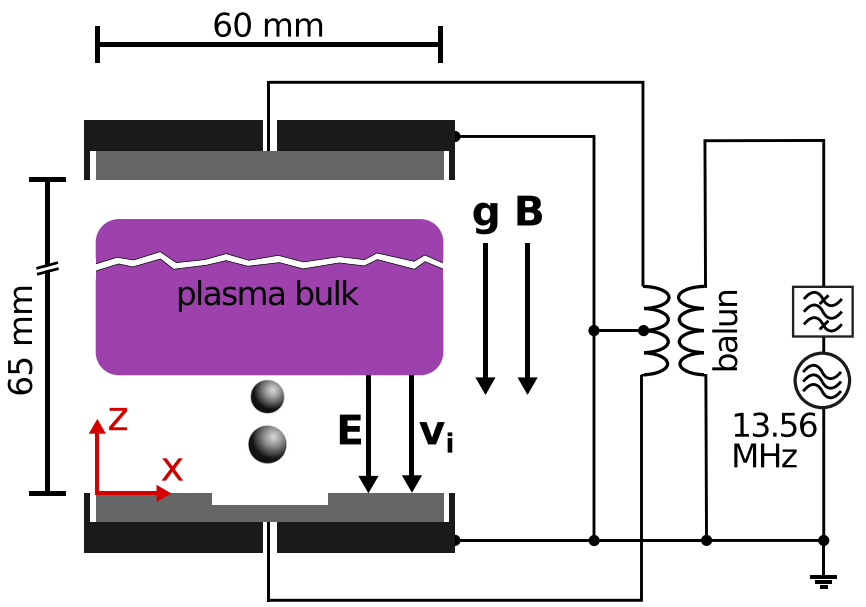

FIG. 1. Sketch of the experimental setup (not to scale): The two shielded electrodes are symmetrically driven via a balun. Particles are confined in the plasma sheath above the lower electrode by an elliptic cavity $(2 \mathrm{~mm}$ depth, $10 \mathrm{~mm}$ semimajor axis, and $6 \mathrm{~mm}$ semiminor axis). The particles are illuminated with a laser and observed using a high-speed camera. A strong magnetic field $B \leq 4 \mathrm{~T}$ is superimposed in the vertical direction. of the mutual repulsion in a particle pair is justified by comparing the weight force $(10-20 \mathrm{pN})$ and the Coulomb force $(0.3 \mathrm{pN})$ for typical values of our setup. Therefore, each particle is tied to its levitation plane, but the position of this plane can be affected by charge reduction in the wake of the other particle.

The particles are illuminated by an expanded laser beam and observed with a high-speed camera. The horizontal confinement of the particles is realized by a cavity in the lower electrode, which has the shape of an elliptic cylinder and whose major semiaxis is aligned with the image plane of the camera. This ensures that the particle pair is aligned with the image plane. In this way, accurate measurements of the interparticle distance are possible when the microparticles are horizontally displaced. In addition, the ellipticity suppresses particle cluster rotations induced by the magnetic field. ${ }^{39}$

The whole plasma device is embedded in a superconducting solenoid, which provides homogeneous magnetic inductions of up to $4 \mathrm{~T}$. Due to plasma instabilities in the magnetic field (see below), new particles can only be inserted into the system at $B=0 \mathrm{~T}$ and the magnetic field has to be increased afterwards.

Magnetized rf-discharges are known to suffer from spontaneous filamentation, ${ }^{40,41}$ which sets in for $B>0.2 \mathrm{~T}$. Filaments are long bright structures that form parallel to the magnetic field lines and propagate through the discharge perpendicular to $B$ (see Fig. 2). In our case, the influence of filamentation effects on the suspended particles is weak for $B<2 \mathrm{~T}$.

Histograms of the positions and velocities of a single microparticle at $B=0.8 \mathrm{~T}$ are shown in Fig. 3. The stochastic motion excited by the filaments causes random velocities of less than $v=7 \mathrm{~mm} / \mathrm{s}$, i.e., kinetic energies of up to $E_{\text {kin }}<4 \times 10^{-17} \mathrm{~J}$. However, the filaments constitute a very efficient heating mechanism, providing kinetic particle temperatures of more than $T_{\text {kin }}=\sigma_{x, z} m \omega_{x, z}^{2} / k_{\mathrm{B}}>10^{8} \mathrm{~K}$, with $\sigma_{x, z}$ the standard deviation of the particle position, $\omega_{x, z}$ the confinement frequencies of the particle in each spatial direction, and $k_{\mathrm{B}}$ the Boltzmann constant.

For $B>2 \mathrm{~T}$, the filamentation instabilities become stronger and disturb the particle confinement. Therefore, the accessible range of magnetic inductions in our experiments is limited to $B<2 \mathrm{~T}$.

\section{B. Characterizing the microparticles}

The glass microballoons used have been differentially sieved to narrow the range of particle radii to $a \in[10,12.5] \mu \mathrm{m}$.

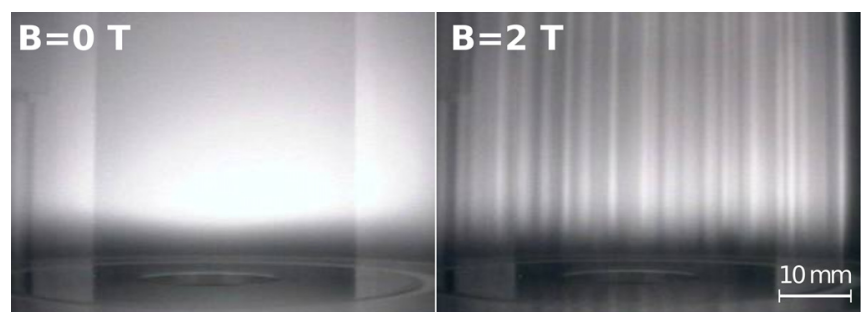

FIG. 2. Side view on plasma and lower electrode with a cavity at $B=0 \mathrm{~T}$ and $B=2 \mathrm{~T}$. At strong magnetic fields, the plasma filaments, long bright structures that propagate through the discharge, occur and tend to disturb the confinement of microparticles. 

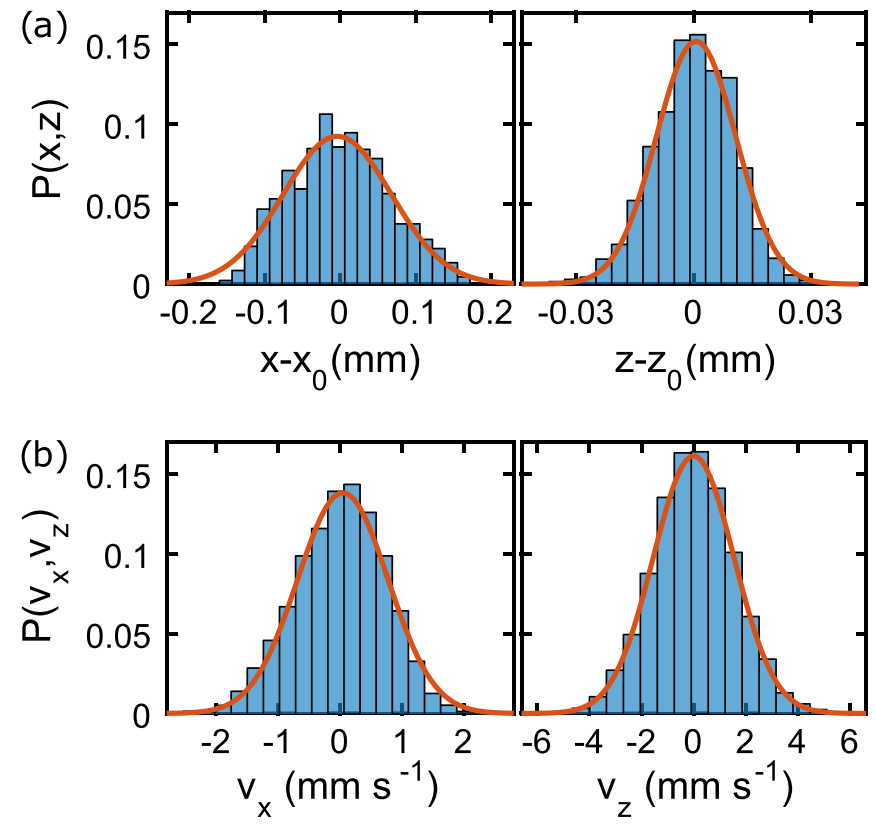

FIG. 3. Histograms of the (a) positions and (b) velocities of a single microballoon at $B=0.8 \mathrm{~T}$ in comparison to a Gaussian distribution (red line). $x_{0}$ and $z_{0}$ are the mean equilibrium positions of the particle.

The advantage of using glass microballoons over the commonly used solid plastic particles is the small effective mass density $\rho=220 \mathrm{~kg} \mathrm{~m}^{-3}$ of the microballoons, resulting in particle masses of $m \approx(0.92 \ldots 1.8) \times 10^{-12} \mathrm{~kg}$. The large size-to-mass ratio alleviates the particle confinement in the presence of weak fluctuations from the filamentation instabilities. The large particle size also improves the observability and results in a larger charge, which guarantees stronger wake effects.

In order to study the wake structure in magnetized plasma, we first need to obtain detailed information on the size and mass of microparticles that form a vertically arranged pair in such a plasma. Vertical pairs require a small mass difference that leads to different levitation heights. If the initial mass difference is not large enough, the particles arrange horizontally.

To determine the size and mass ratio of the two particles, a typical pair has been studied with the phase-resolved resonance method (PRRM). ${ }^{19,42}$ This method is based on the complete evaluation of the dynamical response of the particles to small vertical, periodical perturbations, which are provided by a modulation of the rf power. The particles can be treated as damped harmonic oscillators, with their equation of motion given by

$$
\ddot{\xi}+2 \gamma \dot{\xi}+\omega_{0}^{2} \xi=K \exp (\mathrm{i} \omega t)
$$

with $\xi$ the excursion of each particle from its equilibrium position, $\gamma$ the gas friction coefficient, $\omega_{0}$ the confinement frequency, and $K$ and $\omega$ the amplitude and the frequency of the external driver, respectively. The strength of the PRRM over the traditional evaluation of the amplitude response $e^{4}$ is the full analysis of the complex response function. Using $\xi$ $=A \exp (\mathrm{i} \omega t)$ to solve Eq. (2) leads to the complex amplitude $A$ of the particle oscillation, which is measured as a function of the driving frequency to obtain resonance curves.

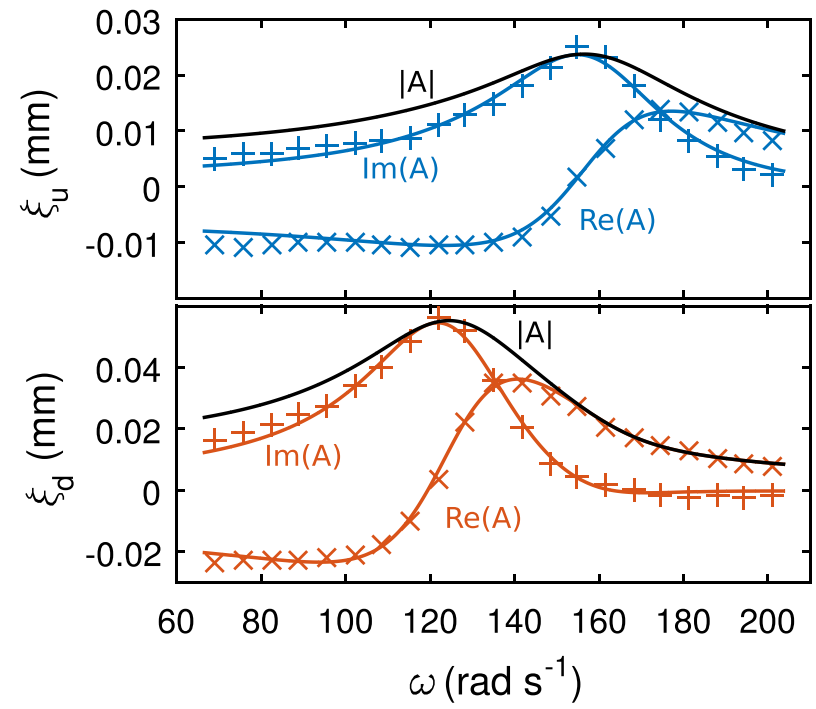

FIG. 4. Resonance curves of an almost horizontally aligned pair of microballoons at $B=1 \mathrm{~T}$. The number of shown data points is reduced by a factor of 3 for clarity. The full lines are the fitted real and imaginary parts, as well as the absolute value of the complex oscillation amplitude $A$.

Here, the initially vertical particle pair has been forced into an almost horizontal state by increasing the magnetic field to $B=1 \mathrm{~T}$ and the rf power to $P_{\mathrm{rf}}=15 \mathrm{~W}$. The underlying mechanism will be discussed in Sec. III B.

The resonance curves of the now horizontal particle pair are shown in Fig. 4. By fitting the real and imaginary parts of the complex amplitude, the eigenfrequencies $\omega_{0}$ and gas friction coefficients $\gamma$ can be determined (Table I). The errors are estimated from synthetic data. ${ }^{43} \mathrm{Using}^{42} \omega_{0} \propto(q / m)^{1 / 2}$ $\propto(a / m)^{1 / 2}$ and $^{44,45} \gamma \propto a^{2} / m$ leads to

$$
\frac{\gamma_{\mathrm{u}}}{\gamma_{\mathrm{d}}}=\frac{a_{\mathrm{u}}^{2} m_{\mathrm{d}}}{a_{\mathrm{d}}^{2} m_{\mathrm{u}}}
$$

and

$$
\frac{\omega_{\mathrm{u}}}{\omega_{\mathrm{d}}}=\left(\frac{a_{\mathrm{u}} m_{\mathrm{d}}}{a_{\mathrm{d}} m_{\mathrm{u}}}\right)^{1 / 2} .
$$

The indices $u$ (upstream) and $d$ (downstream) identify the microparticles in a pair. Rewriting Eq. (4) as

$$
\frac{m_{\mathrm{d}}}{m_{\mathrm{u}}}=\frac{\omega_{\mathrm{u}}^{2}}{\omega_{\mathrm{d}}^{2}} \frac{a_{\mathrm{d}}}{a_{\mathrm{u}}}
$$

and inserting it into Eq. (3) give the size ratio of both particles: $a_{\mathrm{u}} / a_{\mathrm{d}}=0.79 \pm 0.013$. The mass ratio is determined by inserting this in Eq. (5): $m_{\mathrm{d}} / m_{\mathrm{u}}=2.08 \pm 0.031$. Both ratios are close to the outer limits of the particle properties, the particle size $(10 \mu \mathrm{m} / 12.5 \mu \mathrm{m}=0.8)$ and the corresponding particle mass $\left(1.8 \times 10^{-12} \mathrm{~kg} / 0.92 \times 10^{-12} \mathrm{~kg}=1.96\right)$.

TABLE I. Vertical eigenfrequencies $\omega_{0}$ and gas friction coefficients $\gamma$ of an almost horizontally aligned particle pair.

\begin{tabular}{lcc}
\hline \hline & Upstream particle & Downstream particle \\
\hline$\omega_{\mathrm{u}, \mathrm{d}}\left(\mathrm{rad} \mathrm{s}^{-1}\right)$ & $162.7 \pm 0.25$ & $126.9 \pm 0.13$ \\
$\gamma_{\mathrm{u}, \mathrm{d}}\left(\mathrm{s}^{-1}\right)$ & $24.2 \pm 0.36$ & $18.7 \pm 0.13$ \\
\hline \hline
\end{tabular}


As a cross check, the manufacturer information of a mass density of $\rho=220 \mathrm{~kg} \mathrm{~m}^{-3}$ is used to calculate the particle sizes from the gas friction coefficients, ${ }^{44}$ giving $a\left(\gamma_{\mathrm{u}}\right)$ $=(9.7 \pm 0.2) \mu \mathrm{m}$ and $a\left(\gamma_{\mathrm{d}}\right)=(12.3 \pm 0.1) \mu \mathrm{m}$. Again, this fits the outer limits of the sieved particle load.

In summary, by means of resonance measurements, we have characterized the particles that are used to create a vertically aligned pair: The requirement for the mass difference in combination with the small used size interval yields pairs with the upstream (downstream) particle representing the lower (upper) bound of the size distribution.

\section{Simulation of the plasma sheath}

In a second step, we are interested in the plasma parameters in the sheath and the relative position of the microparticles to the sheath edge. We therefore start with studying the plasma parameters in the sheath by PIC simulations. For this purpose, collisional PIC simulations of the rf discharge have been performed with the XPDP1 code ${ }^{46,47}$ at our experimental conditions (electrode gap width, gas pressure, and $\mathrm{rf}$ power) at $B=0 \mathrm{~T}$. XPDP1 is a $1 \mathrm{~d}-3 \mathrm{v}$ code. Because the sheath electric field is parallel to the magnetic field, the sheath conditions are nearly independent of the strength of the magnetic field. We have modified the code by using improved ion cross-sections for elastic and charge-exchange collisions. ${ }^{48}$ The one-dimensional code allows us to determine the potential and density distribution in the plasma discharge and especially in the plasma sheath. It also provides the mean ion velocity at the levitation height of the microparticles - which is a critical parameter for the simulation of wake effects in streaming plasmas.

Figure 5(a) shows the potential $\Phi$, the time-averaged electric field $\langle E\rangle$, and the density profiles $n_{\mathrm{i}, \mathrm{e}}$ of ions and
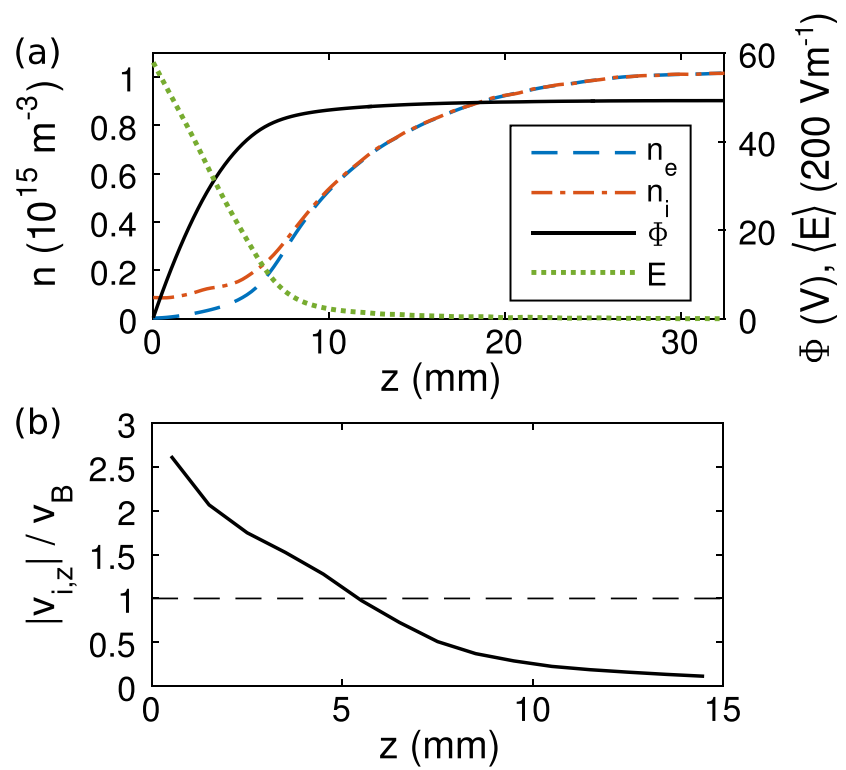

FIG. 5. Results of XPDP1 simulations for a discharge gap of $65 \mathrm{~mm}$, $p=10 \mathrm{~Pa}$, and $U_{\mathrm{rf}}=180 \mathrm{~V}_{\mathrm{pp}}$. (a) Electron density $n_{\mathrm{e}}$, ion density $n_{\mathrm{i}}$, potential $\Phi$, and time-averaged electric field $\langle E\rangle$ as a function of $z$. Due to symmetry, only half of the electrode gap is shown. (b) Mean ion velocity in the $z$ direction in the plasma sheath. Due to the sheath electric field, the ions are accelerated towards the electrode and reach $M=\left|v_{\mathrm{i}, \mathrm{z}}\right| / v_{\mathrm{B}}=1$ at $z=5 \mathrm{~mm}$. electrons from the PIC simulation. A steady plasma bulk is formed in the discharge center, and the quasi-neutrality is fulfilled down to the pre-sheath edge at a distance of $z_{\mathrm{s}}$ $=8 \mathrm{~mm}$, which we define by $n_{\mathrm{es}}=0.9 n_{\mathrm{is}}=3.2 \times 10^{14} \mathrm{~m}^{-3}$.

The ion velocity in the plasma sheath is shown in Fig. 5(b). The ions reach Bohm velocity $v_{\mathrm{B}}$ and become supersonic $M \geq 1$ at $z=5.4 \mathrm{~mm}$. $M$ is the Mach number defined here as the ratio of ion velocity $v_{\mathrm{i}}$ and Bohm velocity $v_{\mathrm{B}}$

$$
M=\frac{v_{\mathrm{i}}}{v_{\mathrm{B}}}=v_{\mathrm{i}} \sqrt{\frac{m_{\mathrm{i}}}{k_{\mathrm{B}} T_{\mathrm{e}}}},
$$

with $k_{\mathrm{B}}$ the Boltzmann constant and $T_{\mathrm{e}}$ the electron temperature. The typical electron temperature is $k_{\mathrm{B}} T_{\mathrm{e}}=1.9 \mathrm{eV}$ in the plasma bulk and $1.6 \mathrm{eV}$ at the sheath edge.

The points where $n_{\mathrm{e}} \neq n_{\mathrm{i}}$ sets in and where $v_{\mathrm{i}}=v_{\mathrm{B}}$ is reached do not coincide, which is characteristic of collisional plasma discharges. ${ }^{49}$

The electron Debye length is often used as a typical scaling length. We choose the Debye length corresponding to the plasma parameters at the pre-sheath edge $\left(z_{\mathrm{s}}=8 \mathrm{~mm}\right)$ as the reference scaling length

$$
\lambda_{\mathrm{De}}=\sqrt{\frac{\epsilon_{0} k_{\mathrm{B}} T_{\mathrm{e}}}{n_{\mathrm{es}} e^{2}}}=0.53 \mathrm{~mm},
$$

with $e$ the elementary charge and $\epsilon_{0}$ the permittivity of free space.

The consistency of the simulational and experimental results can be verified by a PRRM measurement with a single heavy microballoon at the typical discharge properties of $p=10 \mathrm{~Pa}, P_{\mathrm{rf}}=5 \mathrm{~W}$, and $B=0 \mathrm{~T}$. The measurement has been performed with a particle that levitates at $z=4.64 \mathrm{~mm}$. The PRRM measurement yields $\omega_{0}=77.7 \mathrm{rad} \mathrm{s}^{-1}$ and $\gamma$ $=15.9 \mathrm{~s}^{-1}$, resulting in a particle mass of $m=2.1 \times 10^{-12} \mathrm{~kg}$. Using the local ion density at the particle position [Fig. 5(a)] yields a charge number ${ }^{4}$ of the particle of

$$
|Z|=\frac{\omega_{0}^{2} \epsilon_{0} m}{e^{2} n_{\mathrm{i}}}=29000 .
$$

According to Eq. (1), we obtain a time-averaged electric field of $\langle E\rangle=4400 \mathrm{~V} \mathrm{~m}^{-1}$, which agrees with the electric field resulting from the simulations at the particle position $z=4.64 \mathrm{~mm}$ [Fig. 5(a)].

\section{Plasma behavior and particle levitation}

We continue with the experimental description and analysis of the levitation of microparticles in comparison to the development of the plasma glow in the sheath as a function of the magnetic field.

In Fig. 6, the levitation height of a single microballoon is compared with the plasma glow intensity for magnetic inductions $B=(0 \ldots 2)$ T. The plasma glow is measured with a high-sensitivity CMOS camera. The glow intensity is a rough indicator for the time-averaged electron density in the sheath.

The plasma glow is nearly constant at magnetic inductions of $B>0.5 \mathrm{~T}$, and the particle levitation height coincides 


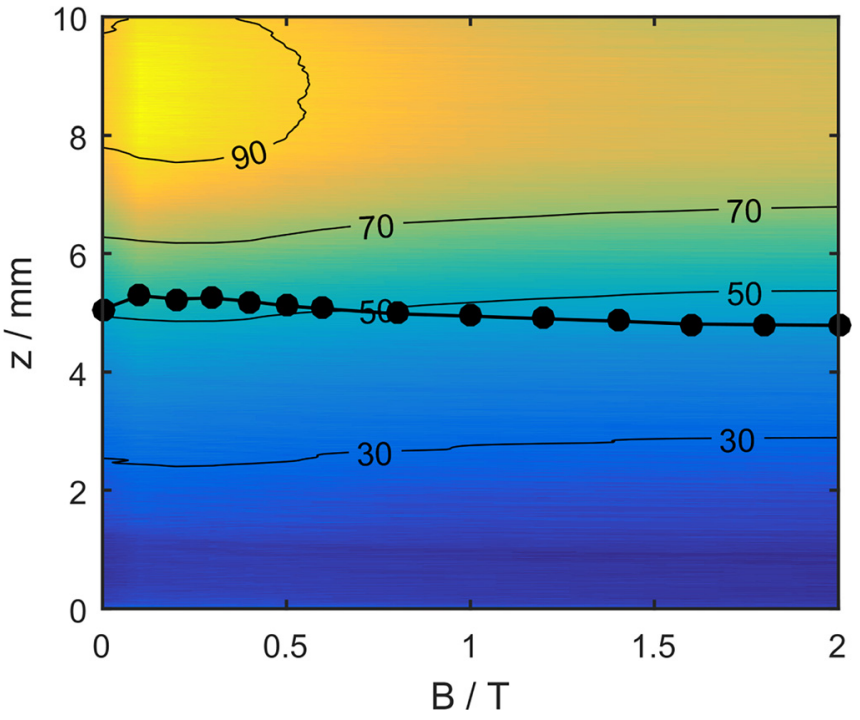

FIG. 6. Lower plasma sheath at $p=10 \mathrm{~Pa}$ and $P_{\mathrm{rf}}=5 \mathrm{~W}$ : time-averaged, normalized plasma glow (color coded with contour lines) and levitation height above the electrode $z_{0}$ of a single $(a \approx 10 \mu \mathrm{m})$ microballoon (circles) as a function of the magnetic induction $B . z=0$ is the position of the electrode surface.

with the $45 \%-52 \%$ intensity contours and shows a slight decrease. The plasma glow indicates that for $B>0.5$, $\mathrm{T}$ an increase in the magnetic field does not lead to changes in the discharge. The global influence of the magnetic field on the electrons, which can be described by the ratio of gyro and plasma frequencies, is quite large already at $B=0.5 \mathrm{~T}\left(\omega_{\mathrm{ce}} /\right.$ $\left.\omega_{\mathrm{pe}}>50\right)$, and so, a further increase in the magnetic field leaves the discharge unchanged.

Hence, it is justified to assume that experiments with pairs of microballoons in this regime will give reliable information about the local physics in the vicinity of the particles: the interaction forces and wakes. Note that the ratio of the particle radius and the electron gyro radius $a / r_{\text {ge }}$ just becomes unity at about $0.6 \mathrm{~T}$, i.e., the electron fluxes on the particle become anisotropic and particle charging is still affected by an increasing magnetic field. ${ }^{36}$

The levitation height in our experiment corresponds to the position, where $v_{\mathrm{i}} \approx v_{\mathrm{B}}$ is reached, as it has been determined in Sec. IIC. Hence, it is reasonable to compare our results with simulations for streaming ions at $M=1$.

\section{PAIR OF MICROPARTICLES IN A STRONGLY MAGNETIZED PLASMA}

\section{A. Change of arrangement at a variation of $B$}

After having studied the properties of single particles, we now explore the behavior of particle pairs in the accessible range of magnetic fields.

Due to small mass differences in combination with wake effects, at $B=0 \mathrm{~T}$, the two particles always levitate in a vertically aligned arrangement. Their absolute positions and relative arrangement are observed, while the magnetic field is slowly increased at $\mathrm{d}|B| / \mathrm{d} t<0.1 \mathrm{~T} / \mathrm{min}$.

This vertical alignment remains unchanged at weak magnetic inductions $B \leq 0.6$ T. Simulations ${ }^{36}$ show that for low magnetizations, there is no significant difference from the unmagnetized case, which has been broadly studied in previous investigations ${ }^{7,9,16,50}$ and hence is well known: The particle arrangement with respect to the flow is stable against changes in the interparticle distance (a decrease in the particle distance can be induced by an increase in the rf power or by means of plasma inherent etching processes in combination with particles of different materials ${ }^{9}$ ). The particle distance is mainly given by the undisturbed equilibrium position of the single particles in the plasma sheath. ${ }^{7,9,50} \mathrm{~A}$ removal of a particle out of a vertically arranged particle pair only leads to small changes in the levitation height of the other particle due to secondary effects as the Coulomb repulsion of the particles and the reduction of the charge of the lower particle in the ion focus of the upper one. ${ }^{7,9}$ The attractive force by the enhanced ion density in the focus region is an important mechanism for the horizontal stability of the vertical particle pair ${ }^{16}$ but plays a minor role in the vertical position of a (downstream) particle due to the strong confinement in the sheath. ${ }^{50}$

Figure 7 shows the evolution of the particle positions in $x$ (horizontal) and $z$ (vertical) directions immediately before a sudden configuration change occurs. The displayed range of magnetic fields corresponds to an observation time of $7 \mathrm{~min}$ and allows detecting precursor events that may initiate the transitions. The increase in the magnetic field [Fig. 7(a)] first and foremost leads to a shift in the stable horizontal position and - as it is known from the study of the single particle (see Fig. 6) - a small descent of the two particles. The horizontal shift takes place on much longer time scales than the filamentation-induced particle oscillations, which appear as noisy broadening of the positions $x$ and $z$. This shift of the horizontal equilibrium position can be related to another form of magnetic field induced plasma instability, presumably point discharges at the edge of the electrode cavity.

At $B_{1}=1.3 \mathrm{~T}$, a sudden change in the particle configuration takes place: the vertical alignment is lost and the

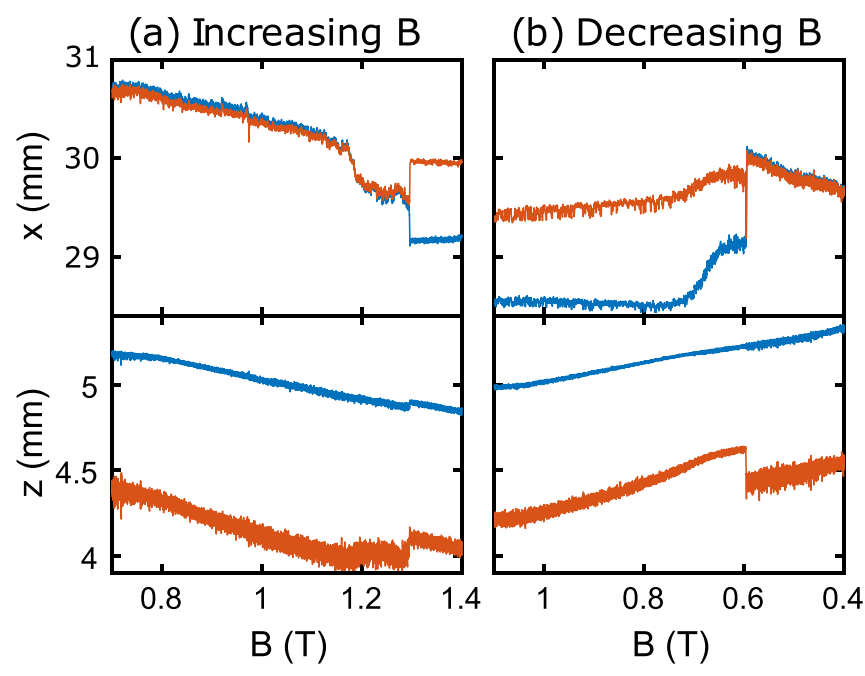

FIG. 7. $x$ and $z$ positions of upper (blue) and lower (red) particles of the same particle pair at $p=10 \mathrm{~Pa}$ and $P_{\mathrm{rf}}=5 \mathrm{~W}$ for (a) increasing and (b) decreasing magnetic fields. Configurational changes occur (a) at $B=1.3 \mathrm{~T}$ and (b) $B=0.6 \mathrm{~T}$. Note that the change in the levitation height of the downstream particle is larger at $B=0.6 \mathrm{~T}$ compared to $B=1.3 \mathrm{~T}$. 
particles switch to an oblique configuration ( $\Delta x$ becomes large). Simultaneously, the levitation height of the lower particle increases. A further increase in $B$ does not change this oblique arrangement, which remains stable over time.

The oblique configuration (independent of $B$ ) is easily understood: The vertical particle distance is mainly given by the different levitation heights from the Millikan condition Eq. (1). The charge of each particle is not affected by the other particle and its wake. The horizontal interparticle distance results from the balance of the repulsive interparticle forces, the restoring electric field force of the horizontal confinement, and the residual weak attractive wake forces.

For decreasing magnetic induction $B$ [Fig. 7(b)], the oblique configuration is preserved down to $B_{2}=0.6 \mathrm{~T}$, where the reverse transition takes place. This magnetic induction is significantly below the former transition point. When the particle pair switches back to the vertically aligned position, an even larger change in the levitation height of the lower particle is found (than in the case of the opposite transition).

Overall, these measurements have been performed with $N=6$ different particle pairs. The absolute magnetic inductions, where the topological transitions occur, are well reproducible in repeated experiments with the same particle pair. For other pairs with different mass ratios, i.e., different initial particle distances, the transition points have different values (see Table II). Nevertheless, a hysteresis width $\Delta B=B_{1}$ $-B_{2} \geq 0.5 \mathrm{~T}$ is always found.

\section{B. Spontaneous change of arrangement at constant B}

A preference for the oblique arrangement at higher field strengths can also be shown in a different way with a constant magnetic field in the range $B \in[0.7,1.1] \mathrm{T}$. The smaller the initial particle distance is the weaker the magnetic induction becomes to establish the vertical alignment. By increasing the rf power to $P_{\mathrm{rf}}=15 \mathrm{~W}$, the interparticle distance of the vertically aligned particle pair is reduced. After a finite time span of $t=(2 \ldots 60) \mathrm{s}$, the lower particle suddenly leaves its meta-stable position. A spontaneous transition to the oblique arrangement takes place, which then remains stable. The transition back to a vertically aligned pair can only be triggered by a strong reduction of the rf power to $P<5$ $\mathrm{W}$, which increases the vertical particle distance. The $\mathrm{rf}$ power is then increased to $P_{\mathrm{rf}}=15 \mathrm{~W}$ again, and the original condition is restored. This mechanism to bring the particles back into vertical alignment by an external variation of the rf

TABLE II. Magnetic inductions $B_{1}$ and $B_{2}$ and the hysteresis width $\Delta B=B_{1}$ $-B_{2}$ for the whole set of measurements with $N=6$ different particle pairs, sorted by $\Delta z_{\text {ref }}$, which is the particle distance of the vertically aligned pair at $B=1 \mathrm{~T}$. The larger the initial particle distance (mass difference), the higher the induction at the transition points $B_{1,2}$.

\begin{tabular}{lcccccc}
\hline \hline & $\# 1$ & $\# 2$ & $\# 3$ & $\# 4$ & $\# 5$ & $\# 6$ \\
\hline$B_{1}(\mathrm{~T})$ & 1.19 & 1.25 & 1.30 & 1.20 & 1.47 & 1.39 \\
$B_{2}(\mathrm{~T})$ & 0.62 & 0.58 & 0.60 & 0.70 & 0.81 & 0.65 \\
$\Delta B(\mathrm{~T})$ & 0.57 & 0.67 & 0.70 & 0.50 & 0.66 & 0.74 \\
$\Delta z_{\text {ref }}(\mathrm{mm})$ & 0.85 & 0.85 & 0.88 & 0.91 & 1.05 & 1.07 \\
\hline \hline
\end{tabular}

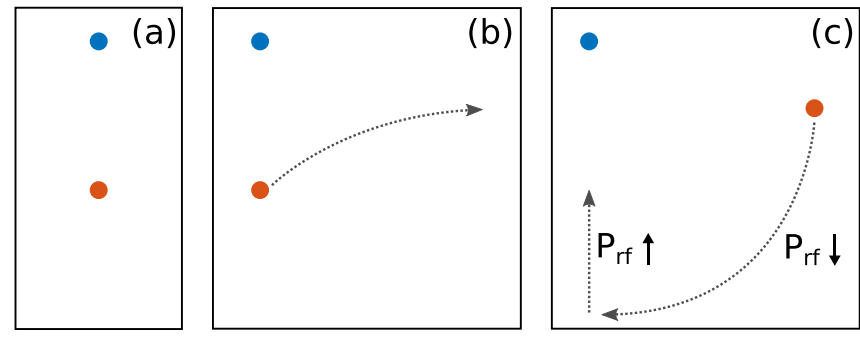

FIG. 8. Sketch of the spontaneous arrangement transition of a particle pair. (a) Initially, the particles are in the vertical state. (b) After a finite time of $t=(2 \ldots 60) \mathrm{s}$, a transition to the oblique configuration sets in (see also Fig. 9). (c) The oblique arrangement is stable over time but can be brought back to vertical by a strong reduction of the rf power which induces an increase in the vertical particle distance. If the rf power is then increased again, the particles are in their initial state and the process restarts.

power is known from previous works. ${ }^{20}$ Here, it is only used to reset the particle configuration.

This cycle is illustrated in Fig. 8. An exemplary spontaneous transition from the vertical alignment to the oblique configuration is shown in Fig. 9.

The merit of the analysis of these spontaneous transitions is that multiple repetitions with an individual particle pair can be performed within a short time, which allows us to analyze the reproducibility of those transitions. The mean
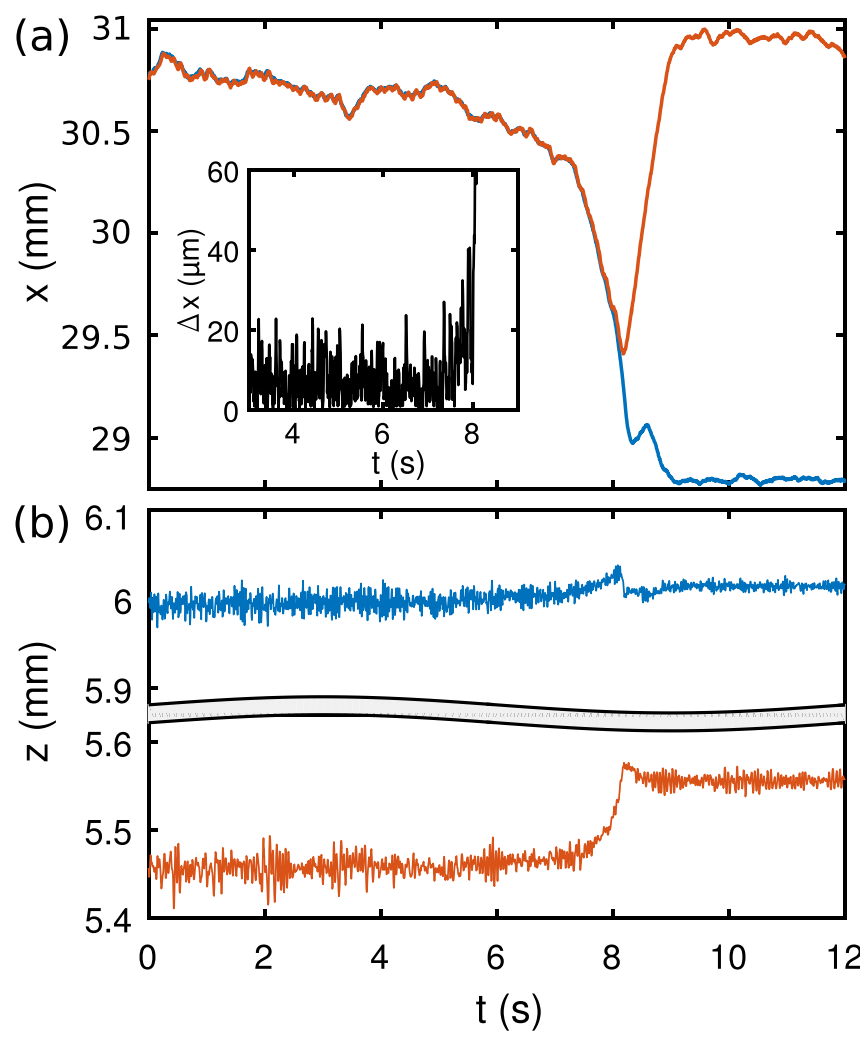

FIG. 9. Spontaneous change in the arrangement of two particles (upper: blue; lower: red) from vertical alignment to the oblique configuration at $B=0.8 \mathrm{~T}$ and $P_{\text {rf }}=15 \mathrm{~W}$. (a) Horizontal positions $x$ as a function of time $t$. Initially, both particles move together away from their equilibrium position. If the deflection is too strong, the lower particle is no longer able to follow the upper particle's movement, and the horizontal component $\Delta x$ of the interparticle distance (shown in the inlay) starts to increase. A distance of at least $\Delta x \geq 30 \mu \mathrm{m}$ has to be exceeded until the transition sets in. (b) Vertical positions $z$ as a function of time $t$. The levitation height of the upper particle stays almost constant, whereas the lower particle is considerably affected. 
TABLE III. Positions of the upper and lower particles in the vertical (aligned) and oblique configuration for the transition process pictured in Fig. 9. Overall, the process has been observed for 22 times for this specific particle pair. $x=30 \mathrm{~mm}$ corresponds to the center of the electrode.

\begin{tabular}{lcc}
\hline \hline & Aligned pos. $(\mu \mathrm{m})$ & Oblique pos. $(\mu \mathrm{m})$ \\
\hline$x_{\mathrm{u}}$ & $30823 \pm 18$ & $28871 \pm 5.7$ \\
$x_{\mathrm{d}}$ & $30814 \pm 17$ & $30997 \pm 10$ \\
$z_{\mathrm{u}}$ & $5995 \pm 1.5$ & $6013 \pm 0.5$ \\
$z_{\mathrm{d}}$ & $5455 \pm 3.0$ & $5557 \pm 0.9$ \\
\hline \hline
\end{tabular}

locations of both particles in both configurations (vertically aligned and oblique) and their standard deviations, which have been determined from 22 single transitions of the same particle pair, are given in Table III. Note that the standard deviation of the average values is smaller than the particle diameter. In both configurations, vertical and oblique, the particles always levitate at the same positions. In all cases, a horizontal particle distance of $\Delta x \geq 30 \mu \mathrm{m} \approx 3 a$ is a threshold that has to be exceeded before the transition sets in.

\section{MOLECULAR DYNAMICS SIMULATIONS OF WAKES}

To explain and understand the experimental observations, we take a closer look at the results of simulations, which made particle charges, ion density distributions in the vicinity of the particles, and the forces on the particles accessible.

For this purpose, we have performed MD simulations of two particles in a streaming plasma environment. The plasma parameters $\left(n_{\mathrm{i}}=2.5 \times 10^{14} \mathrm{~m}^{-3}, k_{\mathrm{B}} T_{\mathrm{e}}=2.5 \mathrm{eV}\right.$, $B=2 \mathrm{~T}$, and $\left.M=1, \mathbf{v}_{\mathbf{i}} \| \mathbf{z}\right)$ have been chosen close to the experimental conditions. The charges of the particles of size $a=12.5 \mu \mathrm{m}$ are calculated self-consistently. For details on the code and its results, see Refs. 16, 36, and 37.

In the collisionless limit ${ }^{36}$ [Fig. 10(a)], ion accumulation in the focus region immediately behind the particle is found. Further downstream, a shadow region (ion depletion) with a negative potential is formed.

In the presence of collisions ${ }^{37}(p=10 \mathrm{~Pa})$ [Fig. 10(b)] and a superimposed dc electric field, the ion focus is elongated and a region of positive space-charge potential is formed downstream. Note that the potential due to the dc

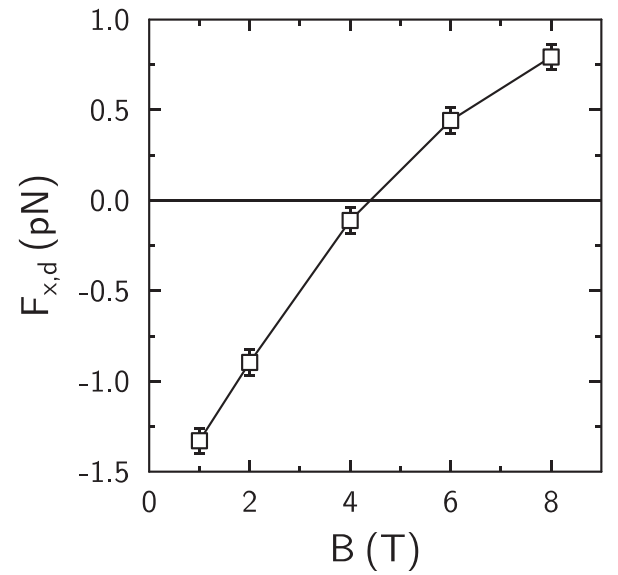

FIG. 11. MD simulation result of horizontal force $F_{\mathrm{x}, \mathrm{d}}$ on the downstream particle as a function of the magnetic induction $B$ at a particle distance of $\Delta x$ $=0.2 \lambda_{\text {De }}$ and $\Delta z=1.0 \lambda_{\text {De. }}$ Positive forces are repulsive (pushing the particle to larger $\Delta x$ ), and negative forces are attractive (pulling the particle to $\Delta x=0)$.

electric field is omitted here to allow the comparison with the collisionless case in Fig. 10(a). In the following, the collisional case is used for further analysis of a particle pair.

Figure 11 shows the restoring horizontal force component $F_{\mathrm{x}, \mathrm{d}}$ on an additional particle in the downstream direction in an almost vertically aligned particle pair $(\Delta x=0.2$ $\lambda_{\mathrm{De}}$ and $\left.\Delta z=1.0 \lambda_{\mathrm{De}}\right)$ as a function of the magnetic induction $B$. An increase in the magnetic field is connected with a decrease in attractive horizontal wake forces, stabilizing the vertical arrangement-as they are known from the unmagnetized system. The forces become repulsive at magnetic inductions of $B>4 \mathrm{~T}$. Note that in the laboratory experiments, the vertical alignment is lost at a smaller magnetic field (Fig. 7). This is not in contradiction to the MD results, which define the confinement of a particle of zero kinetic energy. The downstream particle can leave the confining potential well when its total energy becomes positive. Nevertheless, as discussed in Sec. II A, the random velocities induced by the filamentation instabilities represent a typical kinetic energy of $E_{\text {kin }}<4 \times 10^{-17} \mathrm{~J}$, which is one order of magnitude smaller than the depth of the potential well $\left|W_{\text {pot }}\right|=|q \Phi| \approx|-30000 e \times 50 \mathrm{mV}|=2 \times 10^{-16} \mathrm{~J}$, as it is found in simulations [contour lines in Fig. 10(b)].
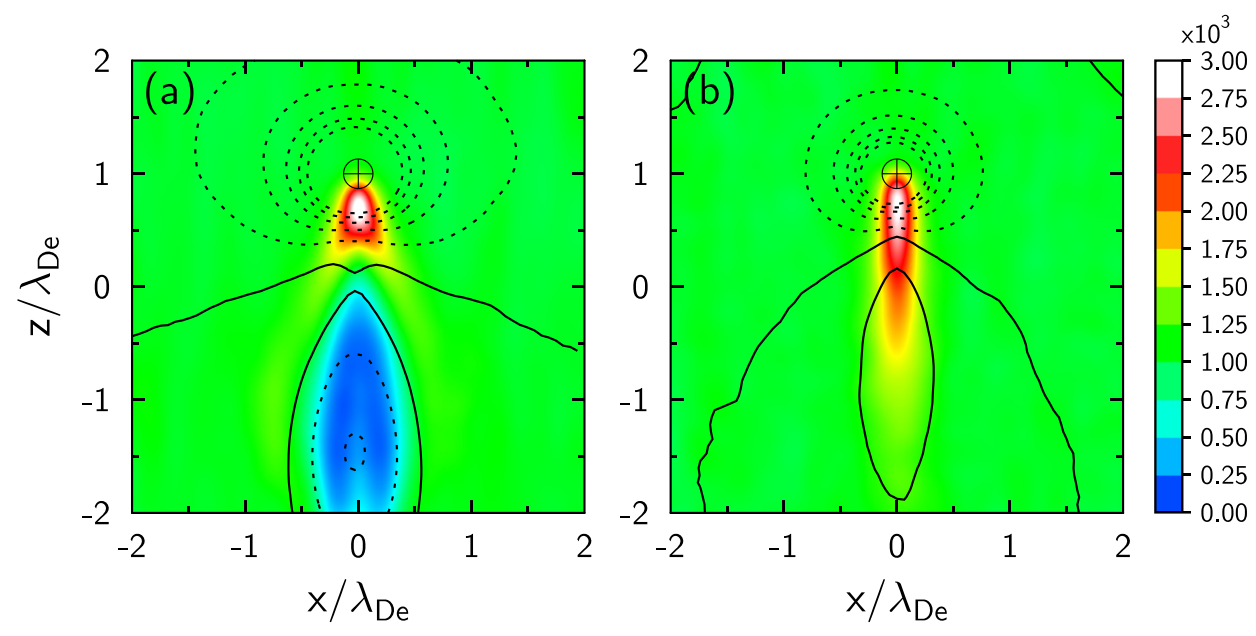

FIG. 10. Density of superions from MD simulations in units of $\lambda_{\mathrm{De}}^{-3}$ (color coded) and potential contours in steps of $50 \mathrm{mV}(\Phi<0$, dotted; $\Phi \geq 0$, full lines) in the vicinity of a single particle at $B=2 \mathrm{~T}$. The particle position is marked with a cross. (a) Collisionless and (b) $p=10 \mathrm{~Pa}$ at an electric field of $E=6260 \mathrm{~V} / \mathrm{m}$. 
All in all, the MD results in the collisional case show that the wake at $B<2 \mathrm{~T}$ (inductions as they are used in the experiment) still exhibits most features of the unmagnetized wake, i.e., an ion density enhancement ("ion focus") and attractive horizontal forces.

To understand the arrangement of the particles, spatial information on the charge of and forces on the lower particle are needed. For this purpose, a set of $10 \times 10 \mathrm{MD}$ simulations with different particle distances $\Delta x$ and $\Delta z$ has been performed. The quantities have been determined, and spatial information has been gained by interpolation.

Figure 12(a) shows how the charge of a second particle is affected by the presence of a particle in the upstream direction at $B=2 \mathrm{~T}$. The particle charge $q_{\mathrm{d}} / q_{\mathrm{d} 0}$ in units of the undisturbed charge $q_{\mathrm{d} 0}$ of a single particle is plotted as a function of the particle separations $\Delta x$ and $\Delta z$. The influence of the ion enhancement in the wake becomes apparent: the closer the lower particle is located to the upper one the more its negative charge is reduced. This effect is extended in the flow direction ( $-z$-direction), where a significant decharging of more than of $10 \%$ can be observed to distances beyond $1 \lambda_{\text {De }}$, but it is limited in the $x$-direction to distances of at most $0.3 \lambda_{\text {De }}$.

This decharging of the downstream particle in the wake and its influence on the levitation height of the lower particle are typical features and known from unmagnetized systems. ${ }^{7,19,27}$ Due to the focus-induced decharging of the particle, its charge becomes more negative when the particle leaves the (horizontally narrow) focus region. Due to the sheath electric field $\langle E\rangle$, a change in the particle charge $q_{\text {new }}$ $=q+\Delta q$ results in an increasing levitation height up to the place, where the Millikan condition [Eq. (1)] is fulfilled again: $(q+\Delta q)(\langle E\rangle+\Delta\langle E\rangle)=m g$. In combination with

$$
\Delta\langle E\rangle=\frac{\mathrm{d}\langle E\rangle}{\mathrm{d} z} \Delta z=\frac{\omega_{0}^{2} m}{q} \Delta z,
$$

this allows us to estimate the change in the particle charge by analyzing the change in its levitation height

$$
\frac{\Delta q}{q}=-\Delta z \frac{\omega_{0}^{2}}{g} .
$$

For a spontaneous transition, as it has been observed in the experiments (Sec. III B, Fig. 9) with $\Delta z=-0.1 \mathrm{~mm}$ and $\omega_{\mathrm{d}}=127 \mathrm{rad} \mathrm{s}^{-1}$ (Sec. II B), this results in $\Delta q / q=16 \%$, which is in good agreement with the charge variation in the simulation results [Fig. 12(a)].

Figure 12(b) shows the MD results of the horizontal force $F_{\mathrm{x}, \mathrm{d}}$ on the downstream particle as a function of its distance to the upper particle at $B=2 \mathrm{~T}$. For vertical separations of $\Delta z \geq 0.5 \lambda_{\text {De }}$, the region close to the symmetry axis of the system $(\Delta x=0)$ is dominated by attractive forces on the downstream particle (as they are already known from Fig. 11). As long as the lower particle is located in this region, it will be pushed back towards the center, resulting in a vertical alignment of the particles. The horizontal extent of this restoring region becomes larger with the increasing vertical distance: from a horizontal distance of $0.4 \lambda_{\text {De }}$ at $\Delta z=0.5$ $\lambda_{\text {De }}$ to $1.2 \lambda_{\text {De }}$ at $\Delta z=1.5 \lambda_{\mathrm{De}}$. A repulsive regime is found above the dashed line in Fig. 12(b).

Overall, the MD results show that two stable arrangements-as found in the experiments-are possible: The attractive forces lead to a vertical alignment (small $\Delta x$ ) of the particles at large $\Delta z$, whereas the repulsive forces at small $\Delta z$ and large $\Delta x$ push the particles apart and-in case of an external confinement-result in an oblique configuration.

In the experiments, the transitions between the two stable configurations show hysteretic behavior. On the one hand, the transition back to the vertically aligned arrangement is based on the increasing attractive wake forces (Fig. 11) when the magnetic field is decreased. On the other hand, the onset of the transition is connected to a previous horizontal approach of the particles [Fig. 7(b)], which is again caused by the shift of the horizontal confinement due to magnetic-field-induced discharge instabilities. Only this horizontal approach allows the downstream particle to re-enter the (a)

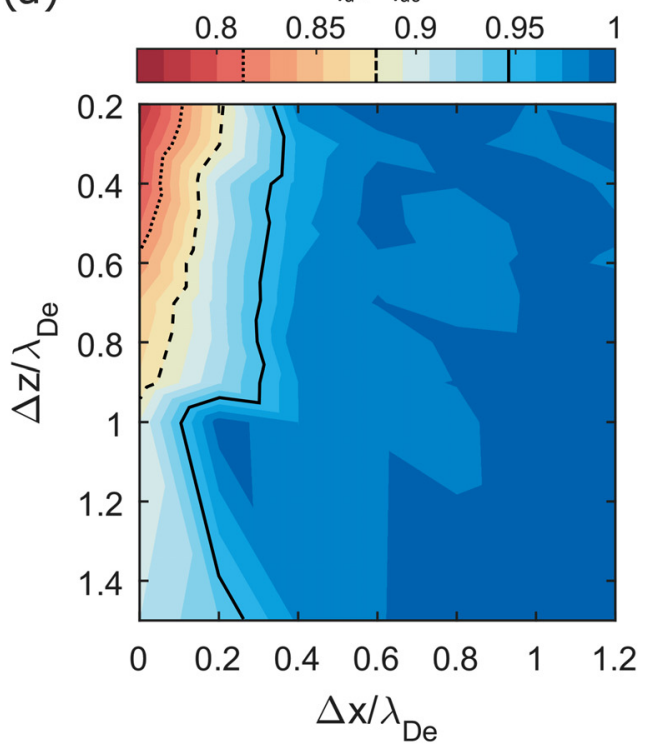

(b)
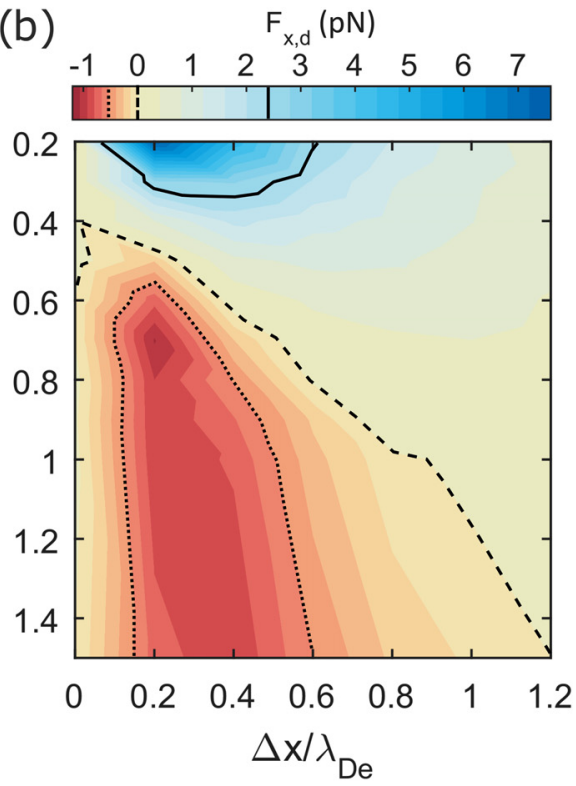

FIG. 12. Spatial structure of wake characteristics determined from interpolation of a set of $10 \times 10$ single MD simulations. (a) Charge variation $q_{\mathrm{d}} / q_{\mathrm{d} 0}$ of a particle in the wake of an upstream particle with respect to the charge $q_{\mathrm{d} 0}=-57370 e$ of a single undisturbed particle as a function of the distance to the upper particle and (b) horizontal force $F_{\mathrm{x}, \mathrm{d}}$ on a lower particle as a function of the distance to the upper particle at $B=2 \mathrm{~T}$. 
narrow attractive wake region, which induces the transition back to the vertical alignment.

We attribute the hysteretic behavior to the nonconservative nature of the forces shown in Fig. 12(b), which includes the charge variation in the wake. Then, the work done on the particle along the path from one configuration to another and back to the initial state is non-zero. Other examples for non-conservative forces by the charge variation are self-excited vortices ${ }^{51-53}$ or self-excited vertical oscillations by delayed charging. ${ }^{54,55}$

\section{CONCLUSIONS}

The arrangement of two microparticles in the plasma sheath with a magnetic field has been studied. Three regimes have been identified: vertical particle alignment at weak magnetic inductions, oblique configurations at the strongest fields, and an intermediate regime, where both arrangements are found-with a preference for the oblique case. This behavior has been investigated by gradually changing the magnetic field and by observing spontaneous transitions at constant parameters. A common feature is the nearly constant levitation height of the upper particle, whereas the lower downstream particle changes its height during the transitions between the configurations.

The existence of two competing arrangements ${ }^{20,24}$ has now also been established for magnetized wakes. Deeper insights are gained by comparing with simulations. MD simulations have been performed at realistic parameters close to the experiment and have shown that the wake of a particle in the collisional regime is still given by a focus-induced ion enhancement [Fig. 10(b)], which provides attractive forces on other microparticles. Furthermore, these attractive forces are weakened by an increasing magnetic field (Fig. 11).

A convergence between the MD simulations and the experiment has been found in the context of decreasing attractive forces, which alleviates the "jump" of the downstream particle out of the focus region and leads to a transition of the particle pair from vertically aligned at low magnetic inductions to an oblique configuration at the highest magnetic fields. What is more, the observed coexistence of two stable particle configurations - oblique and (meta-stable) vertically aligned - at intermediate inductions has been shown by the analysis of (horizontal) forces of a particle pair in MD simulations [Fig. 12(b)].

The combination of MD simulations and PRRM measurements is able to qualitatively explain the exclusive change in the levitation height of the downstream particle during the observed transitions (Figs. 7 and 9), which is due to the decharging of the downstream particle in the ionenhanced wake of the upper particle [Fig. 12(a)]. Further experimental evidence for the modification of the particle wake by magnetic fields was provided by the observation that the change in the levitation height from decharging is much larger at the weak magnetic field [Fig. 7(b)] than at the stronger magnetic field [Fig. 7(a)]. This effect can be attributed to the diminishing wake charge in the ion focus for increasing magnetic induction, as predicted by MD simulations. $^{37}$
Whereas, in simulations, the restoring forces show a change of sign to repulsive forces at $B>4 \mathrm{~T}$ (Fig. 11), vertical alignment is lost at a smaller magnetic field in experiments (Fig. 7). The finding that the vertical alignment in the experiments is lost at significantly lower inductions is not in contradiction to the MD results since the simulations define the confinement of a particle of zero kinetic energy. It has been shown that random velocities induced by the filamentation instabilities cannot trigger the splitting. This is plausible since these disturbances are constantly present and would always trigger transitions immediately and would not allow meta-stable states. We therefore assume that in a rare event, a single strong filament close to the particle pair initiates the splitting.

As an additional supplementary study, the discharge itself has been investigated with self-consistent PIC simulations, i.e., the density profiles, as well as the sheath electric field, and ion flow velocities have been analyzed. On the one hand, this has provided a proper database for comparison with MD simulations. We have shown that the plasma in the vicinity of the microballoons is given by a supersonic $(M \geq 1)$ ion flow. On the other hand, the combination with PRRM measurements, which are used to determine particle properties with high accuracy, demonstrates full consistency of the whole dataset by analyzing the levitation condition of the particles.

In summary, four different approaches-experiments on particle arrangements in the magnetized plasma sheath, MD simulations of the wake structure, resonance measurements on a single particle or pairs, and PIC simulations of the discharge-have been successfully brought together to investigate the influence of a magnetic field on the wake-influenced plasma-particle-system.

\section{ACKNOWLEDGMENTS}

Fruitful discussions with Dietmar Block are gratefully acknowledged. Part of the investigations at Kiel University was financially supported by Deutsche Forschungsgemeinschaft (DFG) within the Transregional Collaborative Research Center SFB-TR 24, Project A2. W.J.M. acknowledges funding by the Research Council of Norway, Project No. 240000.

${ }^{1}$ V. A. Schweigert, I. V. Schweigert, A. Melzer, A. Homann, and A. Piel, Phys. Rev. E 54, 4155 (1996).

${ }^{2}$ K. Takahashi, T. Oishi, K. Shimomai, Y. Hayashi, and S. Nishino, Phys. Rev. E 58, 7805 (1998).

${ }^{3}$ A. Melzer, V. A. Schweigert, and A. Piel, Phys. Rev. Lett. 83, 3194 (1999).

${ }^{4}$ A. Melzer, T. Trottenberg, and A. Piel, Phys. Lett. A 191, 301 (1994).

${ }^{5}$ A. Melzer, V. A. Schweigert, and A. Piel, Phys. Scr. 61, 494 (2000).

${ }^{6}$ W. J. Miloch and D. Block, Phys. Plasmas 19, 123703 (2012).

${ }^{7}$ J. Carstensen, F. Greiner, D. Block, J. Schablinski, W. J. Miloch, and A. Piel, Phys. Plasmas 19, 033702 (2012).

${ }^{8}$ D. Block and W. J. Miloch, Plasma Phys. Controlled Fusion 57, 014019 (2015).

${ }^{9}$ H. Jung, F. Greiner, O. Asnaz, J. Carstensen, and A. Piel, Phys. Plasmas 22, 053702 (2015).

${ }^{10}$ D. Block, J. Carstensen, P. Ludwig, W. J. Miloch, F. Greiner, A. Piel, M. Bonitz, and A. Melzer, Contrib. Plasma Phys. 52, 804 (2012).

${ }^{11}$ P. Ludwig, H. Jung, H. Kählert, J.-P. Joost, F. Greiner, Z. Moldabekov, J. Carstensen, S. Sundar, M. Bonitz, and A. Piel, Eur. Phys. J. D 72, 82 (2018). 
${ }^{12}$ F. Melands $\varnothing$, Phys. Rev. E 55, 7495 (1997).

${ }^{13}$ G. Lapenta, Phys. Plasmas 6, 1442 (1999).

${ }^{14}$ V. R. Ikkurthi, K. Matyash, A. Melzer, and R. Schneider, Phys. Plasmas 15, 123704 (2008).

${ }^{15}$ I. H. Hutchinson, Phys. Plasmas 18, 032111 (2011).

${ }^{16}$ A. Piel, Phys. Plasmas 24, 033712 (2017).

${ }^{17}$ K. J. Brunner, O. Grulke, D. Niemczyk, and T. Klinger, Contrib. Plasma Phys. 54, 802 (2014).

${ }^{18}$ N. J. Prior, L. W. Mitchell, and A. A. Samarian, J. Phys. D 36, 1249 (2003).

${ }^{19}$ H. Jung, F. Greiner, O. H. Asnaz, J. Carstensen, and A. Piel, J. Plasma Phys. 82, 615820301 (2016).

${ }^{20}$ V. Steinberg, R. Sütterlin, A. V. Ivlev, and G. Morfill, Phys. Rev. Lett. 86, 4540 (2001).

${ }^{21}$ O. S. Vaulina, X. G. K. Adamovich, and S. V. Vladimirov, Phys. Scr. 79, 035501 (2009).

${ }^{22}$ I. I. Lisina and O. S. Vaulina, Europhys. Lett. 103, 55002 (2013).

${ }^{23}$ A. A. Samarian, S. V. Vladimirov, and B. W. James, J. Exp. Theor. Phys. 82, 758 (2005).

${ }^{24}$ M. Kroll, J. Schablinski, D. Block, and A. Piel, Phys. Plasmas 17, 013702 (2010).

${ }^{25}$ A. K. Mukhopadhyay and J. Goree, Phys. Rev. Lett. 109, 165003 (2012).

${ }^{26}$ A. K. Mukhopadhyay and J. Goree, Phys. Rev. E 90, 013102 (2014).

${ }^{27}$ W. J. Miloch, M. Kroll, and D. Block, Phys. Plasmas 17, 103703 (2010).

${ }^{28}$ G. A. Hebner, M. E. Riley, and B. M. Marder, Phys. Rev. E 68, 016403 (2003).

${ }^{29}$ G. A. Hebner, M. E. Riley, and B. M. Marder, IEEE Trans. Plasma Sci. 33, 396 (2005).

${ }^{30}$ J. Carstensen, F. Greiner, and A. Piel, Phys. Rev. Lett. 109, 135001 (2012).

${ }^{31}$ M. Nambu, M. Salimullah, and R. Bingham, Phys. Rev. E 63, 056403 (2001).

${ }^{32}$ M. Salimullah, M. Torney, P. K. Shukla, and A. K. Banerjee, Phys. Scr. 67, 534 (2003).

${ }^{33}$ J.-P. Joost, P. Ludwig, H. Kählert, C. Arran, and M. Bonitz, Plasma Phys. Controlled Fusion 57, 025004 (2015).

${ }^{34}$ W. J. Miloch, D. Darian, and M. Mortensen, Phys. Scr. 92, 114006 (2017).

${ }^{35}$ W. J. Miloch, H. Jung, F. Greiner, A. Piel, D. Darian, and M. Mortensen, "Dynamic ion shadows behind finite-sized objects in collisionless magnetized plasma flows," New J. Phys. (in press).
${ }^{36}$ A. Piel, F. Greiner, H. Jung, and W. J. Miloch, "Molecular dynamics simulations of wake structures behind a microparticle in a magnetized ion flow. I. Collisionless limit with cold ion beam," Phys. Plasmas (submitted).

${ }^{37}$ A. Piel, H. Jung, and F. Greiner, "Molecular dynamics simulations of wake structures behind a microparticle in a magnetized ion flow. II Effects of velocity spread and ion collisions," Phys. Plasmas (submitted).

${ }^{38} 3 \mathrm{M}$ Company, Glass Bubbles S22.

${ }^{39}$ J. Carstensen, F. Greiner, L.-J. Hou, H. Maurer, and A. Piel, Phys. Plasmas 16, 013702 (2009).

${ }^{40}$ M. Schwabe, U. Konopka, P. Bandyopadhyay, and G. E. Morfill, Phys. Rev. Lett. 106, 215004 (2011).

${ }^{41}$ E. Thomas, U. Konopka, R. L. Merlino, and M. Rosenberg, Phys. Plasmas 23, 055701 (2016).

${ }^{42}$ J. Carstensen, H. Jung, F. Greiner, and A. Piel, Phys. Plasmas 18, 033701 (2011).

${ }^{43}$ W. H. Press, S. A. Teukolsky, W. T. Vetterling, and B. P. Flannery, Numerical Recipes. The Art of Scientific Computing, 3rd ed. (Cambridge University Press, Cambridge, 2007).

${ }^{44}$ P. S. Epstein, Phys. Rev. 23, 710 (1924).

${ }^{45}$ O. H. Asnaz, H. Jung, F. Greiner, and A. Piel, Phys. Plasmas 24, 083701 (2017).

${ }^{46}$ J. Verboncoeur, M. Alves, V. Vahedi, and C. Birdsall, J. Comput. Phys. 104, 321 (1993).

${ }^{47}$ V. Vahedi, G. DiPeso, C. K. Birdsall, M. A. Lieberman, and T. D. Rognlien, Plasma Sources Sci. Technol. 2, 261 (1993).

${ }^{48}$ A. V. Phelps, J. Appl. Phys. 76, 747 (1994).

${ }^{49}$ K.-U. Riemann, Phys. Plasmas 4, 4158 (1997).

${ }^{50}$ V. Nosenko, A. V. Ivlev, R. Kompaneets, and G. Morfill, Phys. Plasmas 21, 113701 (2014).

${ }^{51}$ O. S. Vaulina, A. P. Nefedov, O. F. Petrov, and V. E. Fortov, J. Exp. Theor. Phys. 91, 1147 (2000).

${ }^{52}$ T. Bockwoldt, O. Arp, K. O. Menzel, and A. Piel, Phys. Plasmas 21, 103703 (2014).

${ }^{53}$ M. Schwabe, S. Zhdanov, C. Räth, D. B. Graves, H. M. Thomas, and G. E. Morfill, Phys. Rev. Lett. 112, 115002 (2014).

${ }^{54}$ S. Nunomura, T. Misawa, N. Ohno, and S. Takamura, Phys. Rev. Lett. 83, 1970 (1999).

${ }^{55}$ A. V. Ivlev, U. Konopka, and G. Morfill, Phys. Rev. E 62, 2739 (2000). 